Brit. Heart F., 1967, 29, 200.

\title{
Electron Microscopic Observations in Alcoholic Heart Disease
}

\author{
CARL S. ALEXANDER \\ From the Departments of Medicine, Minneapolis Veterans Hospital, and University of Minnesota, Minneapolis, \\ Minnesota, U.S.A.
}

The pathological physiology of alcoholic heart disease in man was described as long ago as 1884 (Bollinger, 1884) and little new knowledge has been added since. Recently a report based on electron microscopic examination of eight hearts with alcoholic cardiomyopathy obtained at necropsy described changes in mitochondria, contractile elements, sarcoplasmic reticulum, as well as other subcellular fractions (Hibbs et al., 1965). During the past four years myocardial biopsy using a Menghini needle has been attempted in 60 patients with heart disease of obscure aetiology; all but two were chronic alcoholics. This series of patients was obtained by advising patients suspected of idiopathic heart disease that the nature of their illness was obscure and that new information could be obtained from a tiny bit of heart muscle removed with a special needle, and that such information might prove useful in the prognosis and management of their case. Written consent was obtained from 60 patients before the procedure was carried out, and about one-third of the patients refused their permission. Tissue satisfactory for electron microscopical examination was obtained in about two-thirds of the attempts, and this report describes ultramicroscopical alterations believed to be characteristic, and perhaps even specific, for alcoholic heart disease.

\section{METHOD}

Technique of Biopsy. Biopsy was carried out only in those patients with normal bleeding, clotting, and prothrombin times. The apex was located fluoroscopically and its position marked on the chest wall. Two $\mathrm{cm}$. medial to this point the skin was infiltrated with 1 per cent lidocaine hydrochloride and incised down to the rib with a sharp end of a scalpel blade. If pericardial fluid was suspected, the pericardium was first entered with a blunt 20-gauge needle and the fluid removed. The Menghini needle, $70 \mathrm{~mm} . \times 1.2 \mathrm{~mm}$. or $120 \mathrm{~mm}$. $\times$ $1.2 \mathrm{~mm}$., attached to a $20 \mathrm{ml}$. syringe containing a few Received September 13, 1965. $\mathrm{ml}$. of saline, is advanced toward the myocardium at an angle of 30 degrees to the thorax and directed to the right sterno-manubrial junction. Before entering the myocardium the patient is instructed in breath-holding, and when this is mastered, the needle is advanced obliquely into the myocardium during held expiration. As many as three or four attempts have been made before adequate tissue was obtained. Entry into the cavity of the ventricle occasionally occurs but appears to be of no serious consequence. After the biopsy the patient remains in bed for 24 hours, and pulse, respiration, and blood pressure are checked every 30 minutes for the first two hours, thereafter every four hours for the remainder of the period of observation. A chest X-ray, with the patient on a litter, is obtained the same afternoon of the biopsy, and auscultation of the chest is carried out every three or four hours. If no complications develop the patient may be discharged 48 hours after the biopsy. Antibiotics have not been administered routinely, but adrenocorticosteroids were given to two patients whose pericardial friction rub persisted for more than a few days. The major risk is hæmorrhage, but only on two occasions was withdrawal of blood from the pericardium required to relieve impending tamponade. One patient whose clotting time was erroneously reported as normal bled excessively after biopsy and required a pericardial "window" to prevent tamponade. He died three weeks after biopsy from myocardial failure. Pneumothorax has not been a problem.

Preparation of Tissue. Within less than one minute after biopsy the tissue was immersed in iced 1 per cent osmic acid buffered with veronal acetate at $p \mathrm{H} \mathbf{7 \cdot 4}$, quickly cut into $1 \mathrm{~mm}$. cubes, and allowed to fix for 2 hours at $0-4^{\circ} \mathrm{C}$. After fixation the tissue was washed with the same buffer and dehydrated in 70 and 90 per cent ethyl alcohol followed by acetone, and finally embedded in Vestopal-W. Polymerization was carried out at $60^{\circ} \mathrm{C}$. for 48 hours. Thin sections, $250-400 \AA$, were collected on 200-mesh copper grids coated with Formvar and reinforced with a supporting layer of thin carbon. Sections were then stained with saturated uranyl acetate for two hours and examined in an RCA 3-G model electron microscope. Sections $1 \mu$ thick were examined 200 with the light microscope. 


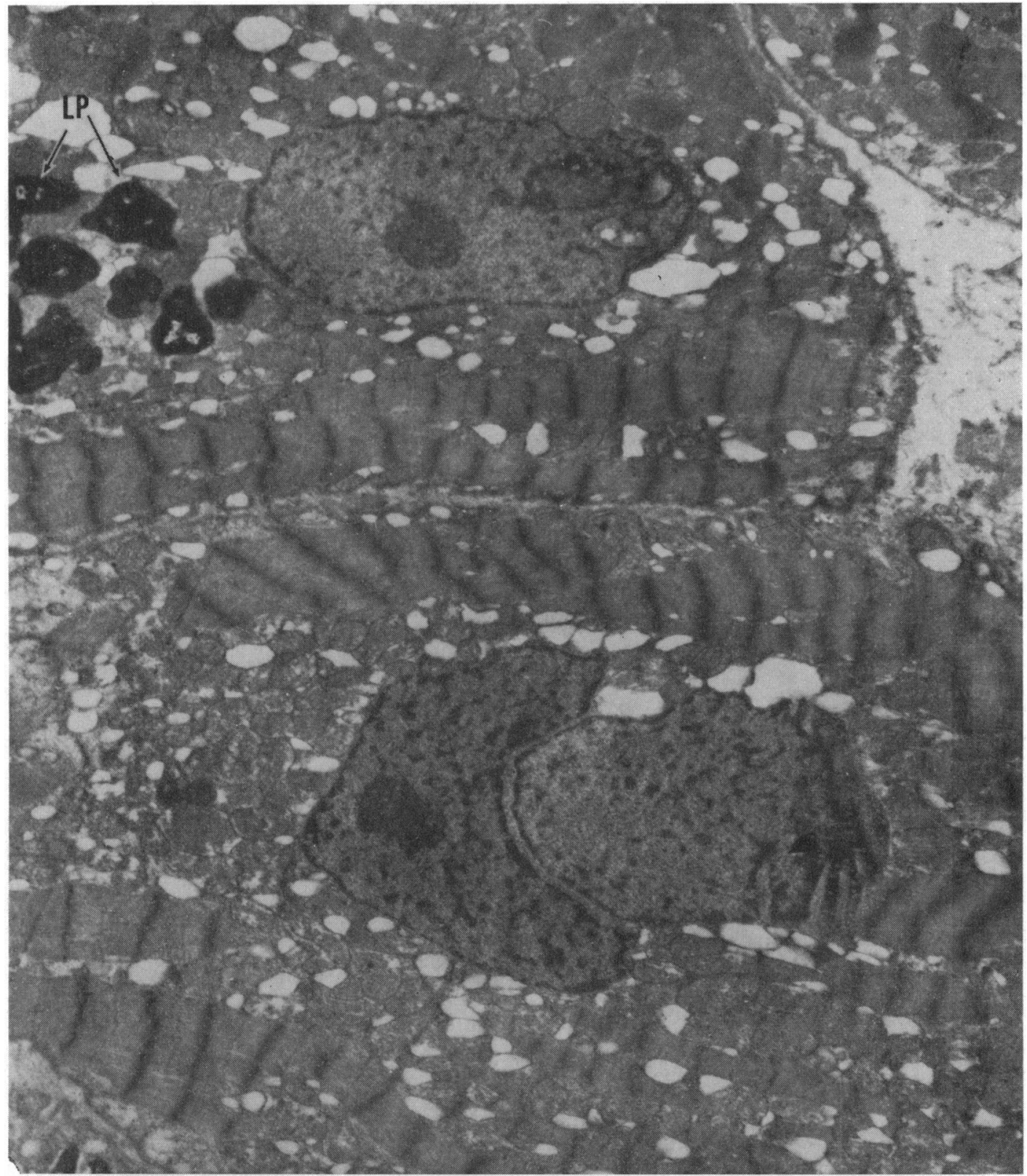

FIG. 1.-Myocardial biopsy of a 60-year-old man with alcoholic heart disease. Myofibrils are in a contracted state and appear reasonably well preserved. Many mitochondria and vacuoles surround the nucleus. Near the nucleus at upper left, lipochrome pigment with stellate clefts can be seen. $(\times 5212$. $) \quad L P=$ lipochrome pigment.

\section{RESULTS}

The more important changes affecting subcellular fractions may be described as follows.

Mitochondria. Mitochondria were increased in number and were so numerous at times that they appeared in sheets between widely spaced myofibrils (Fig. 1). They were enlarged and swollen, and the cristæ were either not well formed, or were disarranged, or degenerating. In some patients the mitochondria contained round dense bodies which 


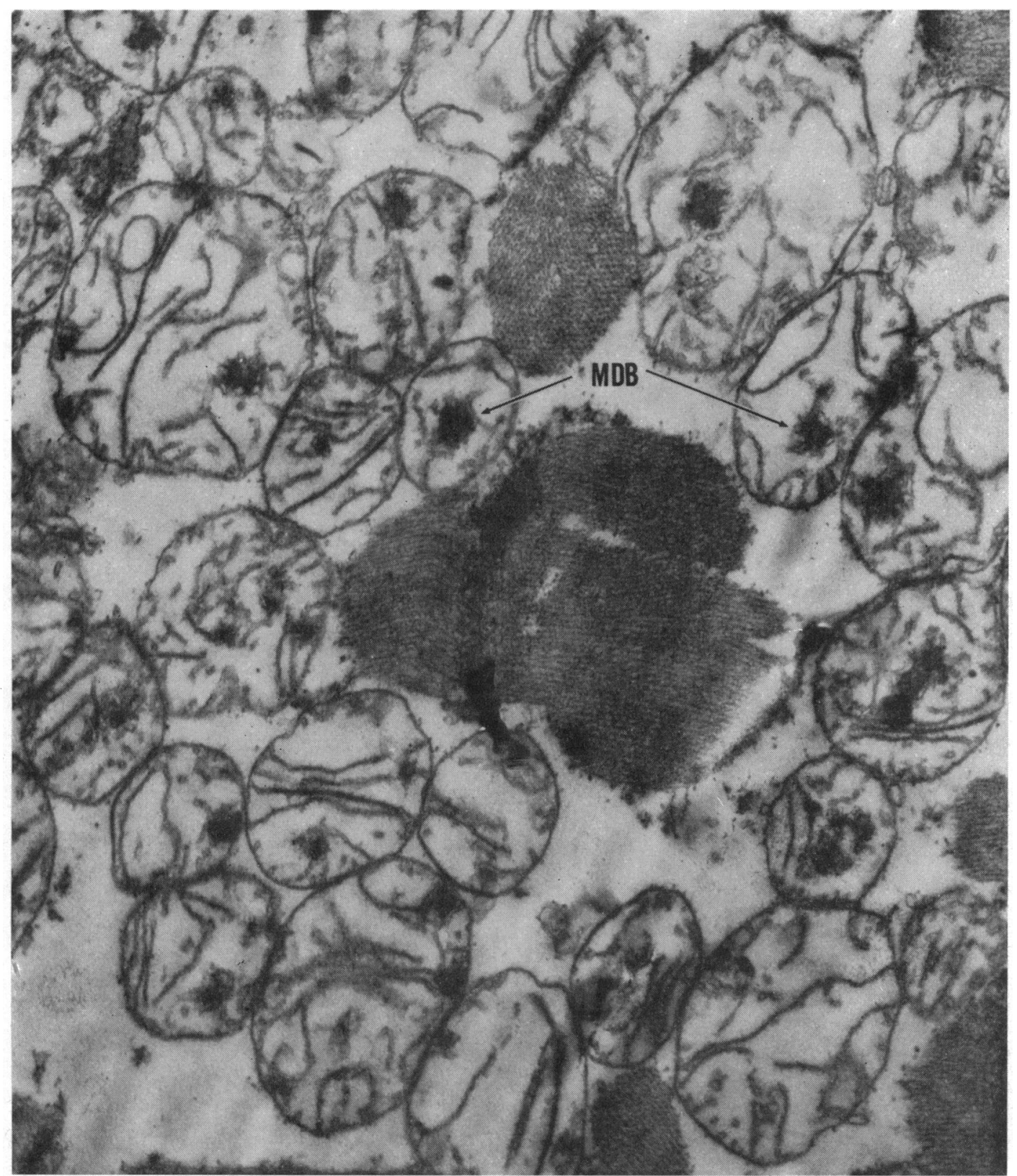

FIG. 2.-Myocardial biopsy of a 42-year-old man with alcoholic heart disease, showing degenerated, short, fragmented myofibrils. Mitochondria are enlarged and swollen. The degenerating cristz are few in number, haphazardly arranged, and are spaced widely apart. Most mitochondria contain round dense bodies also indicative of degeneration. $(\times 26,874) \quad M D B=$. mitochondrial dense bodies.

occasionally were seen outside mitochondria (Fig. 2). They were seen only in the most seriously damaged mitochondria manifested by extensive swelling and loss or degeneration of cristæ. Some mitochondria (Fig. 3) were partially or completely evacuated of their contents ("mitochondrial ghosts") and contributed to the over-all Swiss cheese appearance.

Muscle Elements. The distance between contractile elements was increased either by œdema or 


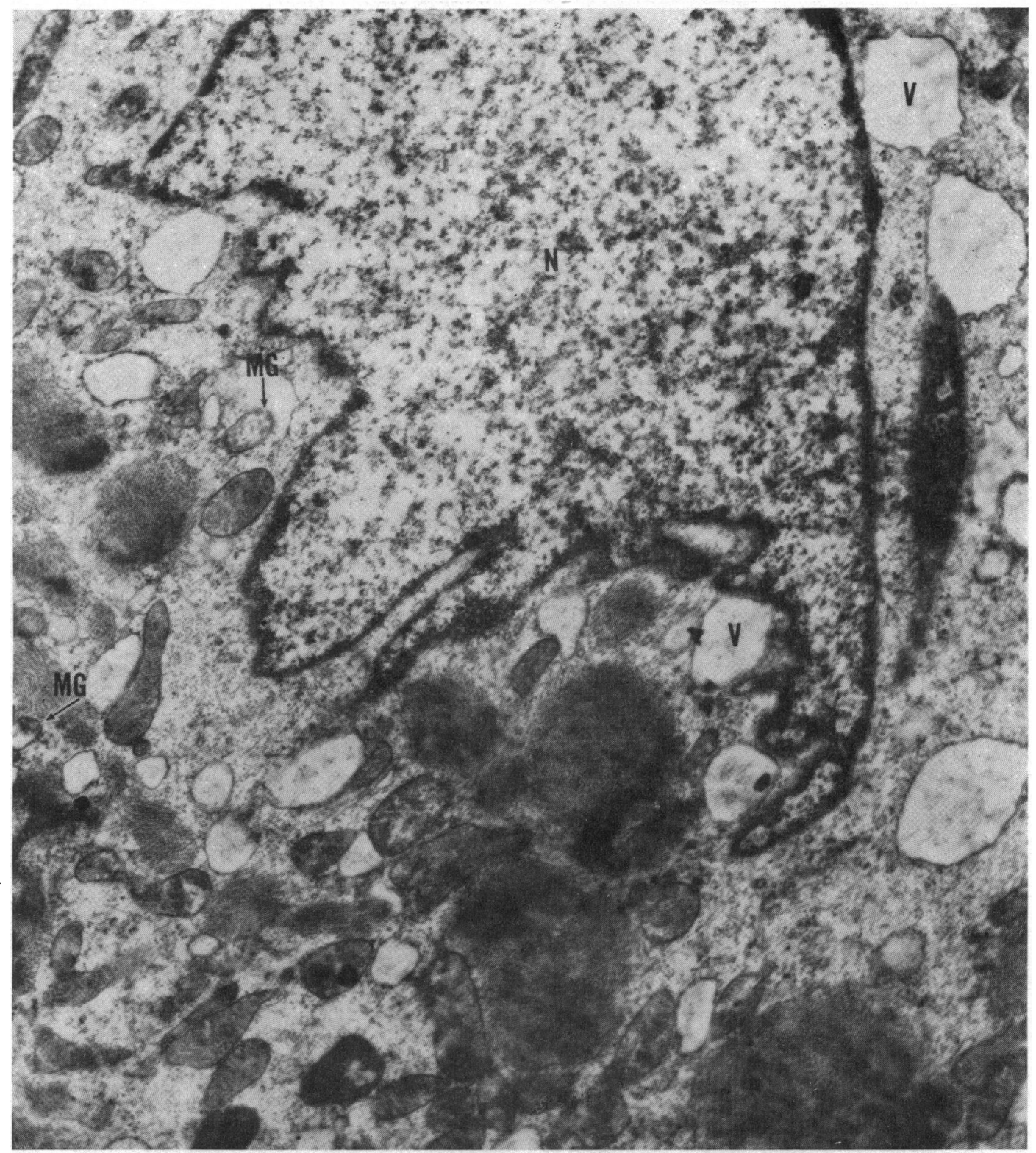

FIG. 3.-Same patient as described in Fig. 2. Short fragmented myofibrils can be recognized only with difficulty. Mitochondria are deformed and vary greatly in size and shape. Large cyst-like vacuoles (V), presumably part of the sarcoplasmic reticulum, present a Swiss cheese appearance. At arrows mitochondria appear moth-eaten, suggesting that they may be forming "mitochondrial ghosts". The serrated nucleus is indented in its lowest portion by vacuoles and myofibrils. $(\times 14,181.) \quad \mathrm{N}=$ nucleus, $\mathrm{V}=$ vacuoles, $\mathrm{MG}=$ "mitochondrial ghosts".

by disappearance of myofibrils, most of which were abnormal in appearance. Myofilaments often stained poorly, especially at the $\mathrm{Z}$ line. In some areas only fragmented short segments were present and appeared to be deprived of the normal anchoring attachments, thus compromising mechanical contractility (Fig. 2 and 3). It was not possible to evaluate muscle hypertrophy by examination with 


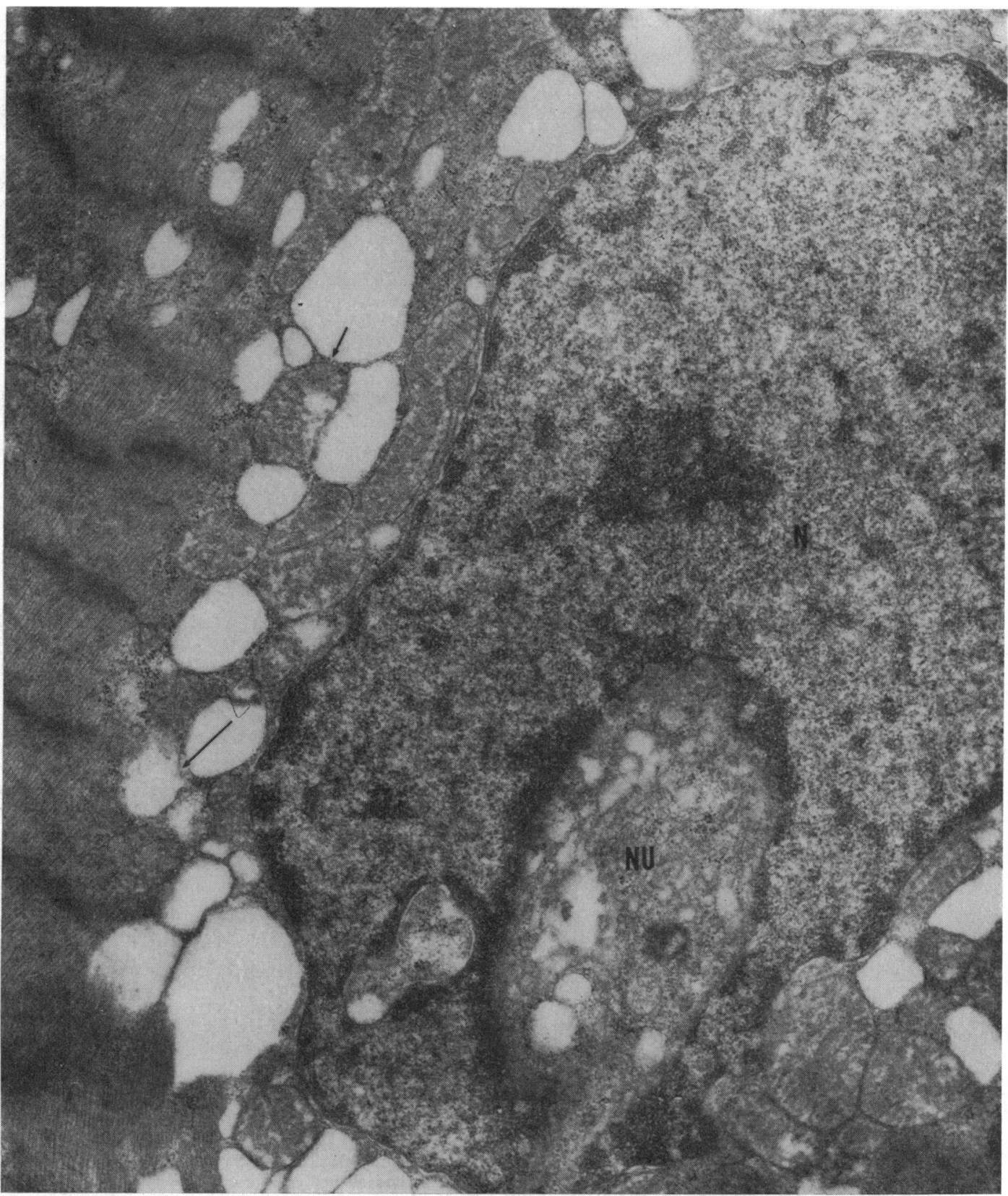

FIG. 4.-Higher magnification of nucleus of same patient as Fig. 1. The nucleus is surrounded by vacuoles and mitochondria, some of which are in the process of evacuating their contents (arrows). Apparent nuclear and even nucleolar phagocytosis is suggested. $(\times 19,969.) \quad \mathrm{NU}=$ nucleolar.

the electron microscope, but this was occasionally recognized under the light microscope.

Sarcoplasmic Reticulum. Sarcoplasmic reticulum seemed to be missing from some areas, but in others it was distended even to cystic proportions, thus also contributing to the Swiss cheese appearance already mentioned. Such vacuoles, which were almost

$$
\text { 西 }
$$


always lined with smooth reticulum, sometimes could not be distinguished from "mitochondrial ghosts".

Nuclei. While it was difficult to judge with certainty, many nuclei appeared to be enlarged and serrated (Fig. 3). The close proximity of vacuoles and mitochondria to the folds of nuclear membrane superficially suggested nuclear phagocytosis (Fig. 3, 4); however, careful examination usually revealed the nuclear membrane to be intact everywhere, indicating that the nuclear membrane was simply being indented by these structures.

Other changes which should be regarded as impressions rather than established observations are occasionally increased lipid and lipochrome pigment and a surprising lack of scarring, except in' the subendocardial region. Lysosomes were few in number. Very likely the quantitative amount of œedema fluid, fat, and glycogen is determined by the state of nutrition and the consumption of alcohol in relation to the time of biopsy. The changes in mitochondria, muscle elements, and sarcoplasmic reticulum in these biopsied hearts are quite similar to those described in the alcoholic hearts examined 1-24 hours after death by Hibbs et al. (1965), and lend additional support to their contention that these changes, at least, are not due to post-mortem autolysis. Similar mitochondrial changes were observed in the liver after alcohol administration (Kiessling et al., 1964; Porta, Bergman, and Stein, 1965; Porta, Hartroft, and de la Iglesia, 1965; Svoboda and Manning, 1964).

\section{Discussion}

It is premature to say that these changes are specific for alcoholic heart disease, or whether or not they may be seen in any failing hypertrophied heart. They have not been seen, however, in the failing or compensated rheumatic hearts of patients at biopsy before being perfused with a pump oxygenator, nor in one patient with non-alcoholic idiopathic heart disease. The latter showed loss of muscle elements and mitochondria with replacement by dense scar tissue and fat. Mitochondrial swelling, damaged and destroyed cristæ, and even round dense bodies have been described in hypoxic dog hearts (Burdette and Ashford, 1963). Whether the mitochondria are actually increased in number or only apparently so as a result of occupying space formerly occupied by contractile elements cannot yet be determined. In myocardial hypertrophy produced by systolic overloading there is no relative increase in mitochondria beyond the enlargement of the cells generally (Meerson et al., 1964). In one study the ratio of mitochondrial protein weight to left ventricular weight and the protein content per mitochondrion were decreased in hypertensive rats as compared to control normotensive rats (Tobian, Severseike, and Cich, 1960). However, increased numbers of mitochondria per unit area ("mitochondriosis") were observed in tissue removed from patients with cardiomyopathy due to left ventricular outflow tract obstruction (Pearse, 1964). These mitochondria were relatively normal in appearance and were unlike those described in patients with alcoholic cardiomyopathy. It seems, therefore, that the relative increase in number of mitochondria may be related to muscular hypertrophy, but this cannot account for their swelling or derangement of cristæ. It is tempting to relate the mitochondrial changes to disturbed metabolism of the cell, due either to alcohol directly or to the accompanying state of malnutrition. In any event, the loss and damage to contractile elements also noted to a lesser extent in obstructive cardiomyopathy (Pearse, 1964) is sufficient to explain the mechanical failure of the enlarged heart.

\section{SUMMARY}

Electron microscopic examination of myocardium obtained by needle biopsy from patients with alcoholic heart disease has revealed striking changes in subcellular elements. In the typical field examined, contractile elements were missing entirely or degenerating, while mitochondria were increased in number. The latter were swollen, while their cristæ were reduced in number or were absent entirely ("mitochondrial ghosts"). In some specimens the sarcoplasmic reticulum was distended to cystic proportions and fat deposits were increased. Some of these changes were probably influenced by timing of the biopsy with respect to removal of alcohol and nutritional supplementation. From preliminary electron microscopic observations made by others in other forms of human heart disease, these changes appear to be characteristic of alcoholic heart disease.

I am grateful to Mrs. Caridad Go for preparation of the specimens for electron microscopy.

\section{REFERENCES}

Bollinger, Prof. (1884). Ueber die Häufigkeit und Ursachen der idiopathischen Herzhypertrophie in München. Dtsch. med. Wschr., 10, 180.

Burdette, W. J., and Ashford, T. P. (1963). Response of myocardial fine structure to cardiac arrest and hypothermia. Ann. Surg., 158, 513.

Hibbs, R. G., Ferrans, V. J., Black, W. C., Weilbaecher, D. G., Walsh, J. J., and Burch, G. E. (1965). Alcoholic 
cardiomyopathy. An electronic microscopic study. Amer. Heart F., 69, 766.

Kiessling, K. H., Lindgren, L., Strandberg, B., and Tobé, U. (1964). Electron microscopic study of liver mitochondria from human alcoholics. Acta med. scand., 176, 595.

Meerson, F. Z., Zaletayeva, T. A., Lagutchev, S. S., and Pshennikova, M. G. (1964). Structure and mass of mitochondria in the process of compensatory hyperfunction and hypertrophy of the heart. Exp. Cell Res., $36,568$.

Pearse, A. G. E. (1964). The histochemistry and electron microscopy of obstructive cardiomyopathy. In Ciba Foundation Symposium. Cardiomyopathies, ed.
G. E. W. Wolstenholme and M. O'Connor, p. 132 Churchill, London.

Porta, E. A., Bergman, B. J., and Stein, A. A. (1965). Acute alcoholic hepatitis. Amer. F. Path., 46, 657.

-, Hartroft, W. S., and de la Iglesia, F. A. (1965). Hepatic changes associated with chronic alcoholism in rats. Lab. Invest., 14, 1437.

Svoboda, D. J., and Manning, R. T. (1964). Chronic alcoholism with fatty metamorphosis of the liver. Mitochondrial alterations in hepatic cells. Amer. $\mathcal{F}$. Path., 44, 645.

Tobian, L., Severseike, O., and Cich, J. (1960). Do mitochondria participate in general cardiac hypertrophy? Proc. Soc. exp. Biol. (N.Y.), 103, 774. 